\title{
Research on medical image denoising
}

\author{
Wang Jinguo ${ }^{1,}$, Wang $\mathrm{Na}^{2, \mathrm{~b}^{*}}$ (corresponding author), Wang rui ${ }^{3, \mathrm{c}}$ \\ ${ }^{1}$ Department of Urology, the First Hospital of Jilin University,China \\ ${ }^{2}$ Department of Anesthesiology, the First Hospital of Jilin University, China \\ ${ }^{3}$ Department of Anesthesiology, the First Hospital of Jilin University, China \\ awangjinguolily@163.com, ${ }^{\mathrm{b}}$ lilyly12345@163.com, ${ }^{\mathrm{C} X i a o b e n 6666 @ 126 . c o m ~}$
}

Keywords: medical image denoising. Anisotropic diffusion. Ultrasonic image. Magnetic resonance image.

\begin{abstract}
Because of the different imaging mechanism, the medical image has many different properties of noise. The existence of these noises affects the observation of medical images and the understanding of the image information. When the noise is serious, the image almost produces the distortion, which makes the image lose the essential meaning of the storage information. Obviously, it is necessary to ensure the image information. The significance of image denoising in addition to improving the accuracy of human visual recognition is still in it is a reliable guarantee for further processing of the image. In this paper, we mainly study some important filtering denoising techniques in digital image processing, which is applied to the real medical image denoising processing.
\end{abstract}

\section{Introduction}

Medical imaging technology is an important part of modern medicine, and has become one of the fastest developing areas in medical technology[1]. It mainly includes medical imaging display technology, medical image analysis processing technology and medical image compression transmission technology[2]. Traditional medical imaging technology is based on physics and modern electronic computer technology. The imaging mechanism is mainly including: projection $\mathrm{X}$ ray imaging, $X$ ray computed tomography, ultrasound imaging, radionuclide imaging, magnetic resonance imaging, infrared imaging and so on. With the further development of computer technology, 3D imaging technology based on holographic technology is becoming more and more widely used, so as to further improve the accuracy of medical diagnosis technology[3].

Medical image noise processing belongs to the image preprocessing stage.From the technical point of view of digital image processing, it belongs to the technical category of image restoration, which has very important significance[4]. Because of the different imaging mechanism, the medical image has many different properties of noise. The existence of these noises affects the observation of medical images and the understanding of the image information. When the noise is serious, the image almost produces the distortion, which makes the image lose the essential meaning of the storage information. In addition to improving the accuracy of human visual recognition, the significance of image denoising is still a reliable guarantee for the further processing of the image. If the feature extraction, registration or image fusion of medical images containing noise are processed, the results can not be satisfactory[5]. Especially for medical image processing, it is necessary to require every step to be as accurate and reliable as possible. So medical image denoising is necessary[6]. 


\section{Medical image noise analysis}

Because the noise generated is randomly, it can only be used to describe the noise in an image, and the noise is considered as a random process, and the whole performance of the noise is used to describe the probability density function of the random process. But in many cases, the probability density function of the noise distribution is very difficult to obtain. So we often use SNR to measure the intensity of the noise.

$$
S N R=\frac{\sigma_{f}^{2}}{\sigma_{n}^{2}}
$$

In which, The $\sigma_{f}^{2}$ and $\sigma_{n}^{2}$ is the variance of signal and noise.

In the experiment, in order to improve the denoising effect of the algorithm, it is often required to add some noise to the image, and then the corresponding algorithm is used to deal with the noise. This is because the noise can be added in advance to know the distribution patterns and the number of features, which can be easily verified by the method of denoising. Image denoising can be used to modify the image histogram, assuming that the image noise to meet a certain distribution. The probability density function is $p(\omega)$. It is not difficult to prove that the random variable $\mathrm{k}$ is the uniform distribution of $[0,1]$. We can get the $\mathrm{k}$ by creating a random function.

$$
k=T(r)=\int_{0}^{r} p(\omega) d \omega
$$

So the corresponding $\mathrm{r}$ is calculated according to the above equation.

\section{Ultrasonic image noise analysis}

Medical image noise can be considered as the texture of the image, which can not reflect the characteristics of the tissues and organs. In the imaging process of ultrasonic image, there is a wide range of speckle noise, which is mainly due to the interaction of ultrasound in the process of imaging. In addition, it has a close relationship with the roughness of the surface of the imaging tissue. Speckle noise can be described by the generalized K distribution, and the probability density function is:

$$
p(x \mid \alpha, v, \eta)=\frac{2 b}{\Gamma(\alpha)}\left[\frac{2 \alpha x}{b \eta^{2}}\right]^{\alpha} I_{0}\left[\frac{v x}{\eta}\right] K_{\alpha-1}\left[b \frac{x}{\eta}\right]
$$

In which, $b=\sqrt{4 \alpha+v^{2}} / \eta, \eta$ is the scaling factor. $v$ describes the coherent part of the echo signal. For the full development of speckle noise, the number of scattered particles is very large, and the generalized $\mathrm{K}$ identities at this time is Rice distribution. At this point $v=0$ the generalized $\mathrm{K}$ is equal to the Rayleigh distribution. From the nature of the noise to the analysis, strictly speaking, the speckle noise contains both multiplicative noise and additive noise. It can be expressed as follow:

$$
\hat{f}=f \cdot n_{m}+n_{\alpha}
$$

But in most of the cases, the effect of additive noise on the image is much less than that of 
multiplicative noise. So the additive noise is ignored, and the speckle noise is a kind of multiplicative noise.

\section{Magnetic resonance image noise analysis}

From the physical view, there are two main sources of magnetic resonance image noise, thermal noise and random noise. Thermal noise is generated mainly from the receiving coil resistance and the thermal motion of the electrons in the imaging object resistance. Magnetic resonance images of random noise source in the impedance of the coil capacitor, in the role of high frequency electromagnetic wave, will produce the induced current in the surface of the object, which will weaken the intensity of electromagnetic waves, causing random noise. In general, the final noise of the magnetic resonance can be considered as the additive noise of the Rayleigh distribution. Its probability density function is:

$$
P(x)=\frac{x}{a^{2}} \exp \left[-\frac{x^{2}}{2 a^{2}}\right]
$$

\section{Denoising principle based on anisotropic diffusion equation}

At all scales, the region should be a priority within the region. According to this criterion, a nonlinear filtering diffusion equation is proposed:

$$
I_{t}=\operatorname{div}(c(t, x, y) \nabla I)
$$

In which, $\mathrm{C}(\mathrm{t}, \mathrm{x}, \mathrm{y})$ is the scale function. in the traditional heat conduction equation $\mathrm{C}(\mathrm{t}, \mathrm{x}, \mathrm{y})=1$, the filtering of the image is obviously not the discrimination ability of the boundary region, the result is bound to lose image details. In the Perona's improvement, it is considered that $c(t, x, y)$ is the norm function of a space $E(t, x, y)$. Reasonably estimate the E. $c(t, x, y)=g(|E|)$. Perona and Malik give the classical selection of $\mathrm{g}(\mathrm{x})$ :

$$
g(x)=\frac{1}{1+(x / K)^{2}}
$$

Assuming $E=\nabla I$, then the diffusion equation is finally obtained:

$$
\left\{\begin{array}{l}
I_{t}=\operatorname{div}(g(|\nabla I|) \nabla I) \\
I_{t=0}=I_{0} \quad\left(\mathrm{I}_{0} \text { is an image of the } \mathrm{t}_{0} \text { moment. }\right)
\end{array}\right.
$$

But then, Catté, Lions, Morel and Coll pointed out that the problem of solving the equation and the stability of the noise. And so on this equation, a little improvement is made. The diffusion equation is as follows:

$$
\left\{\begin{array}{l}
I_{t}=\operatorname{div}\left(g\left(\left|\nabla G_{\sigma} * I\right|\right) \nabla I\right) \\
I_{t=0}=I_{0} \quad\left(\mathrm{I}_{0} \text { is an image of the } \mathrm{t}_{0} \text { moment. }\right)
\end{array}\right.
$$

Then prove the existence and uniqueness of solutions of this equation in the $\sigma>0$, so for this equation in the future application has laid a theoretical basis. Because of the idea of scale space, the 
Perona diffusion equation has the spatial selectivity in filtering the digital image, and the edge preserving property is preserved while filtering out the noise of the image.

\section{References}

[1] David L,Donobo.Denoising by softthresholding[J].IEEE Trans On Information Theory.1995,41(3):617-627.

[2] Coifman R, Donoho.Translation invariant denoising [M]. Springer, New York, 1995:135-150.

[3] Perona $\mathrm{P}$ and Malik J.Scale-space and edge-detection using an isotropic diffusion.IEEE Transactions on Pattern Analysis and Machine Intelligence,

1990,12(7):629-639.

[4] Weickert J.Coherence-enhancing diffusion filtering[J]. International Journal of Computer Vision,1999,31(2-3):111_127.

[5] Loupas T.An Adaptive Weighted Median Filter for Speckle Suppression in Medical Ultrasonic Image[J]. IEEE Trans on Circuits System, 1989, CAS-36(1): 129-135.

[6] Daubechiese I, Lagarias J C.Two-Scale difference equations[J].Math Anal SIAMJ,1991,22:1388-1410. 